\title{
Image Restoration and Topological Optimization
}

\author{
Vaddimukkala Naga \\ Bushanam \\ M.Tech., (IIT) (Ph.D) \\ Sr. Asst. Professor \\ Sir C R Reddy College of \\ Engineering, Eluru - 534 007,
}

A.P. India.

\author{
Dr. G. Samuel Vara Prasad \\ Raju \\ M.Tech. Ph.D \\ Associate Professor, \\ Andhra University, \\ Visakhapatnam, A.P. India.
}

\author{
Vaddimukkala Prasad \\ M.Tech., \\ Bapatla Engineering College \\ Bapatla A.P. India.
}

\begin{abstract}
In this paper, The dissertation deals with the restoration techniques in Image Processing. Tychonoff Regularization method is one of the well known techniques followed in the last two decades. Recently the topological optimization has received the attention of many researchers in the recent past. More surprisingly, Image restoration and segmentation are achieved simultaneously with the help of topological gradient. In this method, the value of the diffusivity $\mathrm{C}$ in the smooth regions, the number of holes drilled in each iteration and the total numbers of holes are the main controlling parameters. A complete Mat lab code has been developed to implement our method. step 1 and 2 will give a brief history of Image Restoration techniques and step 3 will deal with topological optimization techniques and step 4 will discuss the implementation.
\end{abstract}

Keywords: Image Restoration, Tychonoff Regularization, smoothened solution, Finite Element algorithms, Gresh, norm of sum of gradients.

\section{INTRODUCTION}

Digital image processing techniques now are used to solve a variety of problems. Although often unrelated, these problems commonly require methods capable of enhancing pictorial information for human interpretation and analysis.

Geographers use the same or similar techniques to study pollution patterns from aerial and satellite imagery. Image enhancement and restoration procedures are used to process degraded images of unrecoverable objects or experimental results too expansive to duplicate. In archaeology, image processing methods have successfully restored blurred pictures that were the only available records of rare artefacts lost or damaged after being photographed.

In physics and related fields, computer techniques routinely enhance images of experiments in areas such as high-energy plasmas and electron microscopy. Similarly successful applications of image processing concepts can be found in astronomy, biology, nuclear medicine, law enforcement, defence and industrial applications.

Examples of the type of information used in machine perception are statistical moments, Fourier coefficients, and multi-dimensional distance measures.

Typical problems in machine perception that routinely utilize image processing techniques are automatic character recognition, industrial machine vision for product assembly and inspection, military recognizance, automatic processing of fingerprints, screening of x-rays and blood samples, and machine processing of aerial and satellite imagery for weather prediction and crop assessment.

\section{IMAGE RESTORATION}

As an image enhancement, the ultimate goal of restoration techniques is to improve an image in some sense. For the purpose of differentiation, we consider restoration to be a process that attempts to reconstruct or recover an image that has been degraded by using some a priori knowledge of the degraded phenomenon. Thus restoration techniques are oriented toward modelling the degradation and applying the reverse process in order to recover the original image.

This approach usually formulating a criterion of goodness that will yield some optimal estimate of the desired result. By contrast, enhancement techniques basically are heuristic procedures designed to manipulate an image in order to take advantage of the psychophysical aspects of the human visual system. For example, contrast stretching is considered as enhancement technique because it is based primarily on the pleasing aspects it might present to the viewer, whereas removal of image blur by applying a de-blurring function is considered a restoration technique.

Early techniques for digital image restoration were derived mostly from frequency domain concepts. However this chapter focuses on a more modern, algebraic approach, which has the advantage of allowing the derivation of numerous restoration techniques from the same basic principles. Although a direct solution by algebraic methods generally involves the manipulation of large systems of simultaneous equations, we show that, under certain conditions, computational complexity can be reduced to the same level at that required by traditional frequency domain restoration techniques.

\section{PROBLEM DESCRIPTION}

\subsection{Image Restoration}

First we show that there are some similarities between image processing tools and topological optimization methods. Then we will apply topological asymptotic expansion to image restoration.

Let $\Omega \subset R^{2}, v \in L^{2}(\Omega) \mathrm{R}^{2}$, be the noisy image. Let $v=u+\eta$ where $\mathrm{u}$ is the reference image and $\eta$ is the noise. 
A classical restoration is obtained by solving the following boundary value problem.

$$
\begin{aligned}
& -\operatorname{div}(c \Delta u)+u=\operatorname{vin} \Omega \\
& \delta_{n} u=0 \operatorname{on} \Gamma=\delta \Omega
\end{aligned}
$$

Where $\mathrm{c}$ is small. This can be derived by the considering the canonical embedding.

$$
\mathrm{K}: \mathrm{H}^{1}((\Omega) / \mathrm{R}) \rightarrow \mathrm{L}^{2}(\Omega) / \mathrm{R}
$$

$$
\mathrm{U} \rightarrow \mathrm{Ku}=\mathrm{u} .
$$

In order to solve

$\mathrm{Ku}=v$, we consider the minimization problem :

$$
\inf _{\mu \in H^{1}(\Omega) / R} \int_{\Omega}|v-K \mu|^{2} d x
$$

Which is equivalent to

$\mathrm{K} * \mathrm{Ku}=\mathrm{K} * v$.

This is an ill posed problem [3].

\subsection{The Tychonoff Regularization}

The Tikhonov Regularization equation is

$$
\inf _{\mu \in H^{1}(\Omega) / R} \int_{\Omega}|v-K \mu|^{2} d x+\int_{\Omega} c|\mu|^{2} d x
$$

This problem is equivalent to

$\left(\mathrm{K} * \mathrm{~K}+\mathrm{cI}_{\mathrm{d}}\right) \mathrm{u}_{\mathrm{c}}=\mathrm{K} * v$.

The operator

$$
\left(\mathrm{K} * \mathrm{~K}+\mathrm{cI}_{\mathrm{d}}\right)
$$

Has a good property.

\subsection{Variation Formulation}

$\left(\left(K^{*} K+c I_{d}\right) \mu, \omega\right) H^{1}(\Omega) / R=\left(K^{*} v, \omega\right) H^{1}(\Omega) / R \forall \omega \in H^{1}(\Omega) / R$

$(u, \omega) L^{2}(\Omega) / R+(c u, \omega) H^{1}(\Omega) / R=(v, \omega) L^{2}(\Omega) / R \forall \omega \in H^{1}(\Omega) / R$

$$
\int_{\Omega} u(x) \omega(x) d x+\int_{\Omega} c \nabla u(x) . \nabla \omega(x) d x=\int_{\Omega} \nu(x) \omega(x) d x \forall \omega
$$

$$
u-\operatorname{div}(c \nabla u)=v
$$

This is exactly (3.3.1) and the boundary condition $\delta_{n} u=0$ is obtained in a natural way. They the classical restoration method is exactly the Tikhonov regularization applied to the canonical embedding of $\mathrm{H}^{1}$ into $\mathrm{L}^{2}$.

\subsubsection{A Nonlinear Approach}

$$
E(u)=\int_{\Omega}|v-u|^{2} d x+\int_{\Omega} \psi(|\nabla u|) d x
$$

Is equivalent to :

$$
\left\{\begin{array}{c}
-\operatorname{div}(c \nabla u)+u=\operatorname{vin} \Omega, \\
\delta_{n} u=0 \text { on } \Gamma=\delta \Omega
\end{array}\right.
$$

Where

$$
c=\psi^{1}(|\nabla u|) /|\nabla u|
$$

And

$$
\psi(s)=\sqrt{ }\left(1+s^{2}\right)
$$

Is a convenient function

That is

$$
c=\phi(|\nabla u|)
$$

with

$$
\phi(s)=\left(1+s^{2}\right)^{-} 1 / 2
$$

The algorithm:

$$
\begin{aligned}
1-c_{0}= & 1 ; k=0 ; \\
& 2-\text { Compute } \mathrm{u}_{\mathrm{k}} ; \\
& 3-\text { Compute } \mathrm{ck}=
\end{aligned}
$$

$\phi\left(\left|\nabla u_{k}\right|\right)$

$4-$ Go to 2 .

This algorithm recalls the homogenization approach for topological optimization.

3.3.2 Application of the Topological Asymptotic Expansion

$$
\begin{aligned}
& \omega_{p}=x_{0}+\rho \omega \\
& \Omega_{\rho}=\Omega / \bar{\omega} \rho
\end{aligned}
$$

$$
c=\left\{\begin{array}{c}
\operatorname{lin} \Omega_{p}, \\
\in i n \omega_{\rho} . \in>0
\end{array}\right.
$$

To find $\mathrm{c}$ that minimizes:

$$
\begin{aligned}
& j_{c}\left(u_{c}\right)=\int_{\Omega \rho}\left|\nabla u_{c}\right|^{2} d x, \\
& c=\chi \Omega_{\rho} .1+\left(1-\chi \Omega_{\rho}\right) \in .
\end{aligned}
$$

Where $\mathrm{u}_{\mathrm{c}}$ is the solution to

$$
\left\{\begin{array}{c}
-\operatorname{div}\left(c \nabla u_{c}\right)+u=\operatorname{vin} \Omega, \\
\delta_{n} u_{c}=0 \operatorname{on} \Gamma=\delta \Omega
\end{array}\right.
$$




\subsection{The Topological Asymptotic Expansion}

We have that

$$
j(P)-j(o)=J\left(u_{c}\right)-J\left(u_{o}\right)=f(\rho) G\left(x_{o}\right)+o(f(\rho)),
$$

$$
f(\rho)>0
$$

At

$$
\lim _{\rho \rightarrow 0+} f(\rho)=0
$$

If $\omega$ is a ball we have

$$
\left.G\left(x_{o}\right)=-2 \phi\left(\nabla u\left(x_{o}\right) \cdot n\right)\left(\nabla p\left(x_{o}\right) \cdot n\right)-2 \phi \mid \nabla u\left(x_{o}\right) \cdot n\right)\left.\right|^{2}
$$

Where $\mathrm{P}$ is the solution to the ad joint problem

$$
\left\{\begin{array}{c}
-\operatorname{div}(c \nabla p)+p=-\delta_{u} J(u) i n \Omega, \\
\delta_{n} p=0 o n \Gamma .
\end{array}\right.
$$

\section{METHODOLOGY}

\subsection{Implementation}

Input image can be noisy, Excessive smoothing, edges are seemed out, smoothing where gradients are high (edges), reduce $\mathrm{C}$ where gradients are high.

\section{To Choose the Right C}

○ Strategy

- Drill holes in the domain and define $\mathrm{C}$ to be very small inside the holes.

- Set $\mathrm{C}$ equal to some suitable constant outside the holes.

\section{New Idea: Topological Gradient}

Tool from Shape Optimization is following:

○ a domain $\Omega$,

○ a cost functional $\mathrm{J}(\mathrm{u})$,

O With u solutions of a PDE.

An indication on $\mathrm{J}(\mathrm{u})$ will vary by drilling a small hole in the domain at each point $\mathrm{x} 0$. These conditions are applying to the topological gradient.

The flow chart below gives the methodology adopted for restoration. Stopping Criteria.

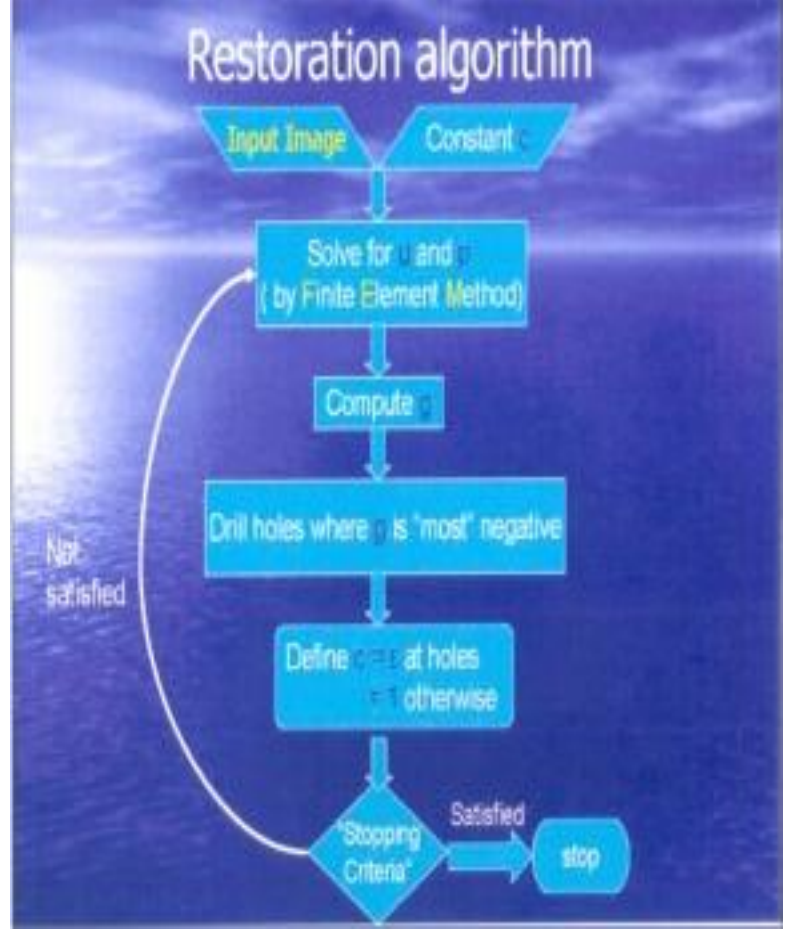

Figure 1

Region homogeneity can be used as stopping criteria:

A filling algorithm is started from a point outside edges. If the filled region pixels are homogeneous, it is accepted as a properly segmented region, and the process is continued with remaining regions. If any region is not acceptable, the whole creation process is continued.

\section{RESULTS AND CONCLUSION}

\subsection{Summary}

The topological gradient method has been adapted to solve restoration and segmentation problems in image processing. Mat lab routines were developed that can handle both gray and RGB colour images. Region filling and merging algorithms were devised and implemented several images with different levels of complexity were processed.

\subsection{Results}

The results obtained by the Image Restoration method in different noise level are given in the following figures:

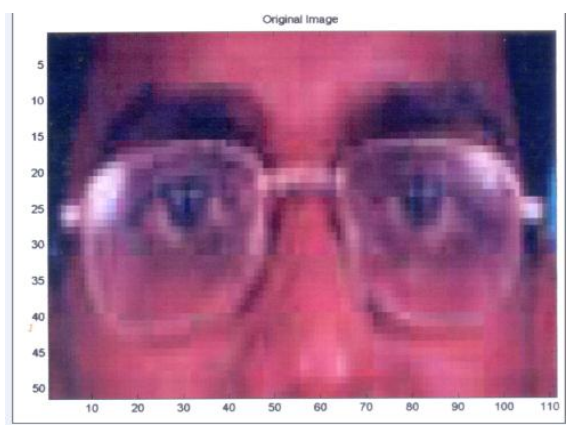

1.1 Original Image 


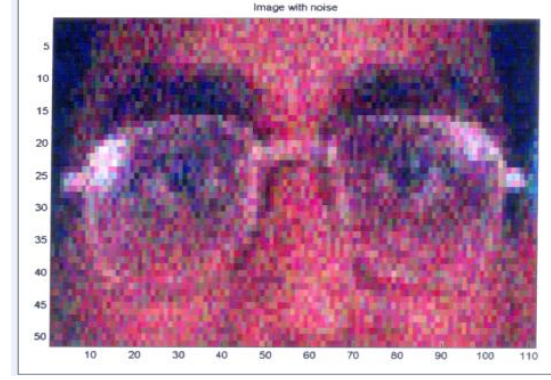

1.2 Image with noise

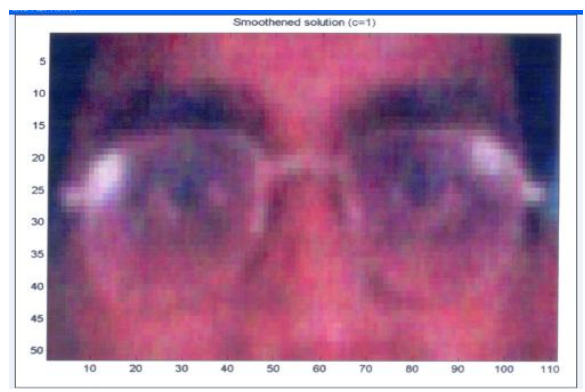

1.3 Smoothened solution $(c=1)$

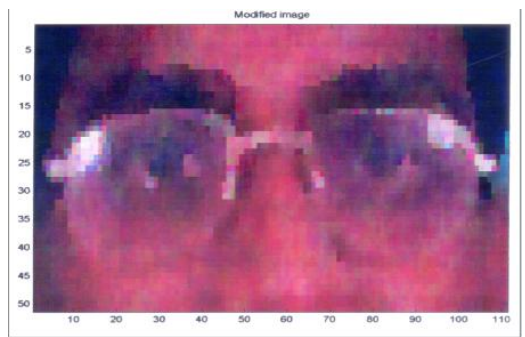

1.4 Modified Image

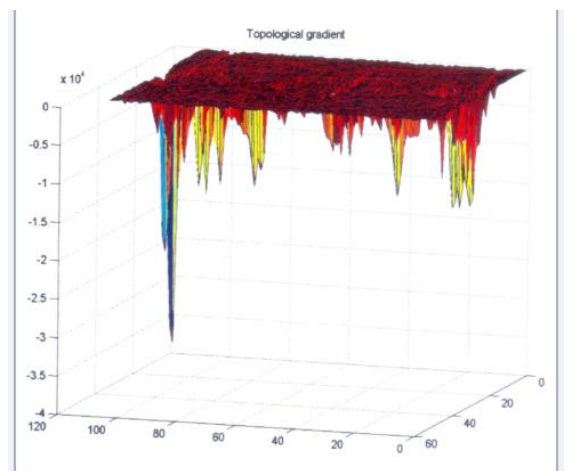

1.5 Topological gradient

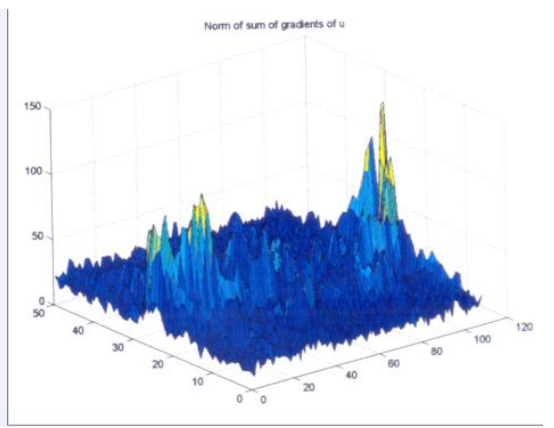

1.6 norm of sum of gradients of $u$

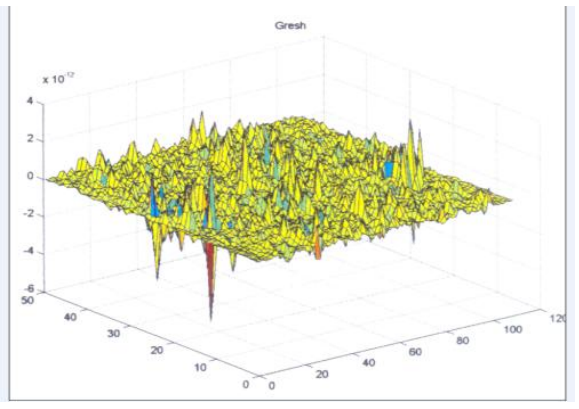

1.7 Gresh

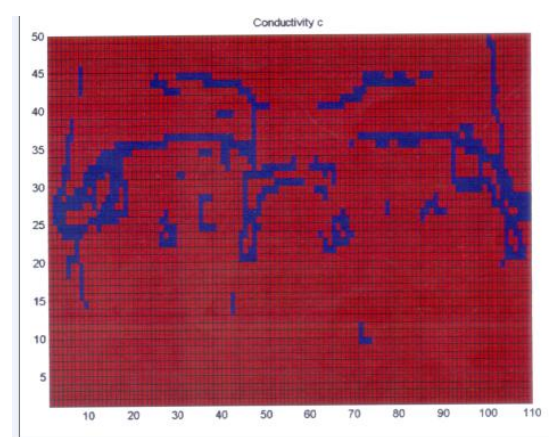

1.8 conductivity $c$

Image Dimensions Width: 350 Height: 287 Colour Depth: 3

The corresponding iteration values:

Iteration $1 \mathrm{~J}=2976660.8691$

Iteration $2 \mathrm{~J}=2792881.8622$

Iteration $3 \mathrm{~J}=2691705.8464$

Iteration $4 \mathrm{~J}=2615838.555$

Iteration $5 \mathrm{~J}=2555238.409$

Iteration $6 \mathrm{~J}=2497211.5443$

Iteration $7 \mathrm{~J}=2447517.0983$

Iteration $8 \mathrm{~J}=2401412.3462$

Iteration $9 \mathrm{~J}=2357630.3415$

Iteration $10 \mathrm{~J}=2313760.0754$

Iteration $11 \mathrm{~J}=2275262.3946$

Iteration $12 \mathrm{~J}=2237846.6716$

Iteration $13 \mathrm{~J}=2202590.2468$

Iteration $14 \mathrm{~J}=2164576.3671$

Iteration $15 \mathrm{~J}=2133058.8258$

Iteration $16 \mathrm{~J}=2100687.2751$

Iteration $17 \mathrm{~J}=2067939.0246$

Iteration $18 \mathrm{~J}=2037216.6449$

Iteration $19 \mathrm{~J}=2008226.5865$

Iteration $20 \mathrm{~J}=1977003.9915$ 


\section{CONCLUSION}

The topological gradient method has been very successful for image restoration and segmentation. Image restoration and segmentation are achieved simultaneously the value of the diffusivity $\mathrm{C}$ in the smooth regions, the number of holes drilled in each iteration and the total number of holes are the main controlling parameters.

\subsection{Future Work}

Different cost functional, Use cracks and anisotropic diffusivity, Develop new stopping criteria, Implement morphological filters to narrow edges.

\section{REFERENCES}

[1] Gonzalez R.C and woods R.E., Digital image processing, Addison Wesley, [2010].

[2] Kornprobst, p.etal. Nonlinear operations in Image Restoration, Preprint [2009].

[3] Masmoudi.M and Samuel A., Image Processing using Topological optimization, preprint [2009].

[4] Scerra, J.: Image Analysis and Mathematical Morphology, vol.2, Academic press, New York, Mc Graw -Hill, New York,(2010).

[5] Skolowski.J and Zochowski.A. Optimality condition for simultaneous topology and shape optimization, SIAM J. Control and optimization.42 (2008), 1198-1221.

[6] Bates, R.H.T., and McDonnell, M.J., 2011, Image Restoration and Reconstruction, Clarendon Press, Oxford.
[7] Jain, A.K., 2010, Fundamentals of Digital Image Processing, Prentice-Hall.

[8] G. Wang, M. W. Vannier, M. W. Skinner, M. G. P. Cavalcanti, G. Harding: Spiral CT image deblurring for cochlear implantation. To appear in IEEE Trans. on medical imaging.

\section{AUTHORS PROFILE}

Vaddimukkala Nagabushanam has completed his M.Tech., in Computer Science and Engineering from Indian Institute of Technology, (IIT) Madras. He is pursuing Ph.D. in Andhra University, (A.U) Visakhapatnam. His research interest includes Image Processing, Data Mining and Data Warehousing etc. He is currently associated with Sir C R Reddy College of Engineering, ELURU, West Godavari District A.P.India. affiliated to Andhra University. He is an active fellow of Computer Society of India (CSI). He is also member of IEEE International conferences.

Vaddimukkala Prasad is pursuing his M.Tech, in Computer Science and Engineering from Bapatla Engineering College, Bapatla, Nagarjuna University (N.U) Guntur A.P. India.His research interest includes Image Processing, Data Mining and Data Warehousing etc. He is an active fellow of Computer Society of India (CSI). $\mathrm{He}$ is also member of IEEE International conferences. 